\title{
LA PRODUCCIÓN CIENTÍFICA Y LA FORMACIÓN DE LOS DOCENTES DE CIENCIAS DE LA INFORMACIÓN DE LA FACULTAD POLITÉCNICA DE LA UNIVERSIDAD NACIONAL DE ASUNCIÓN'
}

THE SCIENTIFIC PRODUCTION AND THE FORMATION OF THE DATA SCIENCES OF THE INFORMATION OF THE FACULTAD POLITÉCNICA DE LA UNIVERSIDAD NACIONALDE ASUNCIÓN

${ }^{1}$ Emilce Noemi Sena Correa

Universidad Nacional de Asunción

\section{Correspondência}

${ }^{1}$ Emilce Sena Correa

Universidad Nacional de Asunción

San Lorenzo, Paraguay

Email: esena@pol.una.py

ORCID: http://orcid.org/0000-0002-

$\underline{6543-6126}$

Submetido em: 10-03-2017

Aceito em: 31-05-2017

Publicado: 12-06-2017

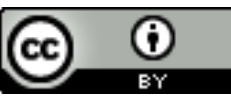

JITA: AC. Relationship of LIS with other fields

${ }^{1}$ Este estudio formó parte del Proyecto Comportamiento Métrico del Desarrollo Disciplinar de las Ciencias Bibliotecológica y de la Información en Iberoamérica. 
RESUMEN: El estudio se propone determinar los factores que condicionan la productividad científica y la relación con la formación académica de los docentes de la carrera de Ciencias de la Información de la Facultad Politécnica de la Universidad Nacional de Asunción, entre los años 2009-2013. El mismo parte de la implementación de un sistema de cuestionarios anonimos, encaminados a la obtención de información y de indicadores sobre las potencialidades de cada país en investigación y docencia. El sistema consta de cuestionarios de tres tipos: cuestionario institucional, cuestionario de recursos humanos y el cuestionario de proyectos de investigación. Las variables en estudio fueron: investigadores, docentes, proyectos de investigación, producción científica, funciones, investigación, indicadores bibliométricos de producción y comunicación científica. Los resultados revelaron que: que en su mayoría el plantel docente de grado son docentes paraguayos $(81 \%)$, y la baja productividad, se ve directamente relacionada con este segmento de la población. Esta situación coincide en general con el modelo de universidad que predomina en Paraguay, que es la "transmisora de conocimiento", en lugar de la "generadora del mismo". Es decir, las actividades docentes y administrativas prevalecen por encima de la labor investigativa; la falta de una adecuada política de incentivo, desestimula la investigación.

PALABRAS CLAVES: Producción científica, Docentes de bibliotecología, Facultad Politécnica-Universidad Nacional de Asunción.

ABSTRACT: The study aims to determine the factors affecting scientific productivity and the relationship with the academic training of professors in the career of Information Sciences at the Facultad Politécnica de la Universidad Nacional de Asunción, between the years 2009-2013. The same part of the implementation of a system of questionnaires aimed at obtaining information and indicators on the potential of each country in research and teaching. The system consists of three types of questionnaires: institutional questionnaire, human resources questionnaire and questionnaire research projects. The variables studied were: researchers, professors, research projects, scientific production, functions, research, bibliometric indicators of scientific production and scientific communication. The results revealed that: that most of the teaching staff are Paraguayans grade professors $(81 \%)$, and low productivity, is directly related to this segment of the population. This situation generally agrees with the university model that prevails in Paraguay, which is the "transmitter of knowledge" instead of "generating the same". Thus teaching and administrative activities take precedence over the investigative work; the lack of an adequate policy incentives, discourages research.

KEYWORDS: Scientific production, Professor of librarianship, Facultad Politécnica-Universidad Nacional de Asunción. 


\section{INTRODUCCIÓN}

La calidad de la educación universitaria se encuentra relacionada con la práctica de la investigación, con el objeto del fomento de la cultura investigativa como también la producción científica donde tanto los docentes,como los equipos de investigación se agrupan para desarrollar actitudes, propiciando intercambios de saberes mediante proyectos de investigación acordes con las líneas de investigación que acuerdan las universidades.

La cultura científica se debe promover desde el aula, y fortalecer y sostener a través de la promoción e incentivo a los investigadores que desarrollan sus líneas de investigación y como resultado se obtiene la producción científica, y la visibilidad de la institución,como de los investigadores.

La investigación científica, dentro de un plan curricular se debe entender más que como un curso teórico, como un curso que se debe aprender haciendo. No se puede comprender, que un DOCENTE no realice INVESTIGACIÓN.

Los docentes que imparten cursos de investigación científica, deben guiar al estudiante en dicho desarrollo, éstos deberían haber realizado investigación previamente, es decir, han debido publicar artículos originales en revistas científicas indexadas.

El caso de los países sudamericanos, la producción científica en el ámbito bibliotecológico es baja, posiblemente debido a los limitados recursos económicos y humanos para la investigación, la baja cultura científica y la deficiente formación en investigación.

Sin embargo, en el país los esfuerzos para motivar a los investigadores a realizar investigación, ha surgido desde el Consejo Nacional de Ciencia y Tecnología, CONACYT, que desde el 2011, creo el Programa Nacional de Incentivo para Investigadores, PRONII, que viene impulsando el desarrollo de la investigación y ha cultivado en los más jóvenes el interés por la investigación científica.

Pero se debe resaltar los problemas financieros por lo que pasa las Universidades públicas, principalmente van a seguir presentes a lo largo de las próximas décadas, e incluso es probable que se agraven porque siempre el recorte se da al presupuesto de la Universidad, afectando principalmente, al sector de la investigación. De ahí que sea necesario que las universidades procuren el acceso a otras vías de financiación, como la empresarial. No se discute que un entorno competitivo, los recursos económicos se asignarían en función de la calidad docente y de investigación.

Cuando se habla de inversión, el Paraguay se encuentra en situación desfavorable según Duarte Masi (2015), porque solo invierte en investigaciones el 0,08\% del PIB, lo que

\begin{tabular}{l|l|} 
v.15 & n.3 \\
\hline
\end{tabular}

p.518-532
set./dez. 2017 
le deja muy por debajo de la media regional, siendo que los países desarrollados invierten más del $1 \%$ del PIB en investigación.

Por qué es relevante la acción:enseñanza/investigación? Un estudio de Sancho Gil (2001)señala los siguientes factores como favorecedores de la transferencia:

- La actividad investigadora lleva a la mejora de la enseñanza. Es más, los académicos no pueden ser buenos, sin hacer investigación, aunque un buen investigador puede no ser un buen docente.

- Algunas de las infraestructuras conseguidas a través de proyectos de investigación también se utilizan para actividades de enseñanza.

Las actividades de investigación contribuyen a poner al día al currículum.

- $\quad$ Si los cursos se relacionan con el perfil investigador del profesorado, la relación es favorable.

Por lo expuesto el rol de las instituciones de educación superior es fundamental para la formación de profesionales idóneos y con capacidades adecuadas para brindar respuestas a la sociedad; los planes de estudio deben poseer lineamientos homogéneos interinstitucionales, revisados y actualizados periódicamente de acuerdo a los requerimientos del mercado laboral y los avances de la ciencia y la tecnología.

\section{EL DESARROLlo ACADÉmico de LA BiBlotecología Y CIENCIAS DE LA INFORMACIÓN EN PARAGUAY}

La enseñanza de la Bibliotecología a nivel universitario en Paraguay data de 1971, año en que se crea la Escuela de Bibliotecología, dependiente del Rectorado de la Universidad Nacional de Asunción. En el año 1987, por Resolución No 2418 la Escuela de Bibliotecología pasa a depender de la Facultad Politécnica. En Paraguay, la Universidad Nacional de Asunción es la única institución, que cuenta con la carrera a nivel universitario, existen otras instituciones privadas, pero solo ofrecen cursos a nivel de diplomado.

A nivel de postgrado, en el 2010 se habilita la Maestría en Ciencias de la Información con tres énfasis: Gerencia de la Información en Medios de Comunicación, Tecnologías de la Información y la Comunicación en Unidades de Información y,Ciencia, Tecnología y Sociedad.

En el 2012, se crea el Grupo de Investigación en Ciencias de la Información, GICI, dependiente también de la Facultad Politécnica. Estas iniciativas pretenden que la Bibliotecología y la Ciencias de la Información, se fortalezcan a nivel nacional y la visibilidad internacional. 
En el 2013, se rehabilita el Curso de Especialización en Gestión Documental y Administración de Archivos, con un plan totalmente actualizado, dando continuación al que se había iniciado en el 2006.

Todos los cursos forman parte del Programa de Postgrado en Ciencias de la Información, dependiente del Departamento de Investigación, Postgrado y Extensión de la Facultad Politécnica. Para el desarrollo de los módulos, se cuenta con el concurso de especialistas nacionales y extranjeros con renombrada trayectoria profesional.

En este transitar, existe colaboración de los pares internacionales, pertenecientes a prestigiosas universidades de América Latina y de Europa.

\section{MATERIALES Y MÉTODOS}

Este trabajo forma parte del Proyecto: Comportamiento Métrico del Desarrollo Disciplinar de las Ciencias Bibliotecológica y de la Información en Iberoamérica, coordinado por el Dr. Salvador Gorbea Portal, Investigador Titular IIBI-UNAM, cuyo objetivo ha sido identificar las potencialidades de docencia e investigación en América Latina, para lo cual se realizaron los diagnósticos nacionales de todos los países.

La información principal para el desarrollo de esta investigación parte de la implementación de un sistema de cuestionarios en línea de carácter nominal, encaminados a la obtención de información y de indicadores sobre las potencialidades de cada país en investigación y docencia. El sistema de cuestionarios, consta de tres tipos: cuestionario institucional, cuestionario de capital humano y el cuestionario de proyectos de investigación.

Los cuestionarios anonimos fueron desarrollados en un sitio Web para facilitar su llenado desde cualquier país participante, página web: http://cuib.unam.mx/ gorbea/observatorio.htm.

Las variables en estudio fueron: investigadores, docentes, proyectos de investigación, producción científica, docencia, investigación, indicadores bibliométricos de producción y comunicación científica, que se encuentra en el Observatorio de Indicadores Científicos, del Instituto de Investigaciones Bibliotecológicas y de la Información (IIBI) de la Universidad Nacional Autónoma de México (UNAM). 
Tabla 1. Relación de variables seleccionadas, según valores

\begin{tabular}{|l|l|}
\hline \multicolumn{1}{|c|}{ Variables de Potencialidades } & \multicolumn{1}{|c|}{ Variables Bibliométricas } \\
\hline Recursos Humanos & Producción Cientifica \\
\hline Cargo: & Tipología Documental: \\
\hline$\cdot$ Docentes & $\cdot$ Artículos \\
\hline$\cdot$ Investigadores & $\cdot$ Libros \\
\hline Grado: & $\cdot$ Capítulos de libros \\
\hline$\cdot$ Doctorado & $\cdot$ Ponencias \\
\hline$\cdot$ Maestría & $\cdot \cdot$ Tesis \\
\hline$\cdot$ Licenciatura & Lugar de Publicación: \\
\hline Proyectos delnvestigación: & $\cdot$ Nacional \\
\hline$\cdot$ En Curso & $\cdot$ Extranjero \\
\hline$\cdot$ Terminados & Fuente de Indización: \\
\hline Programas Docentes: & $\cdot$ Base de Datos Especializada \\
\hline$\cdot$ Licenciatura & $\cdot$ Indice de Citas \\
\hline$\cdot$ Maestría & Idioma dela Publicación: \\
\hline$\cdot$ Doctorado & $\cdot$ Español \\
\hline$\cdot$ Otro & $\cdot$ Inglés \\
\hline Matrícula de Alumno: & $\cdot$ Portugués \\
\hline$\cdot$ Licenciatura & $\cdot$ Francés \\
\hline$\cdot$ Maestría & Origen de publicación de las Revistas Fuente: \\
\hline$\cdot$ Doctorado & $\cdot$ Nacional \\
\hline$\cdot$ Otro & $\cdot$ Extranjera \\
\hline Año & Año \\
\hline Identificador dela Institución & Identificador de la Institución \\
\hline
\end{tabular}

Fuente: Propuesta de un indicador para medir el comportamiento del desarrollo disciplinar de las Ciencias Bibliotecológica y de la Información en instituciones académicas, Gorbea Portal, Piña Pozas. 
Tabla 2. Relación de indicadores seleccionados según tipo para cada Institución

\begin{tabular}{|c|c|}
\hline Indicadores de Potencialidades & Indicadores Bibliométricos \\
\hline $\begin{array}{l}\text { - Recursos Humanos por año, según cargo que se } \\
\text { ocupe }\end{array}$ & $\begin{array}{l}\text { - Producción científica por año, según tipología } \\
\text { documental }\end{array}$ \\
\hline $\begin{array}{l}\text { - Recursos Humanos por año, según el último grado } \\
\text { obtenido }\end{array}$ & $\begin{array}{l}\text { - Producción científica por año, según lugar de } \\
\text { publicación }\end{array}$ \\
\hline - Proyectos de Investigación por año, según tipología & $\begin{array}{l}\text { - Artículos científicos por año, según fuente de } \\
\text { indización }\end{array}$ \\
\hline $\begin{array}{l}\text { - Programas docentes por año, según el nivel } \\
\text { académico impartido }\end{array}$ & $\begin{array}{l}\text { - Producción científica por año, según idioma de } \\
\text { publicación }\end{array}$ \\
\hline $\begin{array}{l}\text { - Matrícula de Alumnos por año, según el nivel } \\
\text { cursado }\end{array}$ & $\begin{array}{l}\text { - Artículos científicos por año, según el origen de } \\
\text { la revista }\end{array}$ \\
\hline $\begin{array}{l}\text { - Valor Promedio de Recursos Humanos según el } \\
\text { cargo que ocupan }\end{array}$ & $\begin{array}{l}\text { - Valor Promedio de la Producción científica según la } \\
\text { tipología documental }\end{array}$ \\
\hline $\begin{array}{l}\text { - Valor Promedio de los Recursos Humanos, según el } \\
\text { último grado obtenido }\end{array}$ & $\begin{array}{l}\text { - Valor Promedio de la Producción cientifica según el } \\
\text { lugar de publicación }\end{array}$ \\
\hline $\begin{array}{l}\text { - Valor Promedio de Proyectos de Investigación } \\
\text { según su tipología }\end{array}$ & $\begin{array}{l}\text { - Valor Promedio de artículos científicos según su } \\
\text { fuente de indización }\end{array}$ \\
\hline $\begin{array}{l}\text { - Valor Promedio de Programas docentes según el } \\
\text { nivel académico impartido }\end{array}$ & $\begin{array}{l}\text { - Valor Promedio de la Producción científica según el } \\
\text { idioma de publicación }\end{array}$ \\
\hline $\begin{array}{l}\text { - Valor Promedio de Matrícula de Alumnos por año } \\
\text { según el nivel cursado }\end{array}$ & $\begin{array}{l}\text { - Valor Promedio de artículos científicos según el } \\
\text { origen de la revista }\end{array}$ \\
\hline $\begin{array}{l}\text { - Estructura porcentual de Recursos Humanos según } \\
\text { el cargo que ocupan }\end{array}$ & $\begin{array}{l}\text { - Estructura porcentual de la Producción científica } \\
\text { según la tipología documental }\end{array}$ \\
\hline $\begin{array}{l}\text { - Estructura porcentual de Recursos Humanos según } \\
\text { el último grado obtenido }\end{array}$ & $\begin{array}{l}\text { - Estructura porcentual de la Producción científica } \\
\text { según el lugar de publicación }\end{array}$ \\
\hline $\begin{array}{l}\text { - Estructura porcentual de Proyectos de Investiga- } \\
\text { ción según la tipología }\end{array}$ & $\begin{array}{l}\text { - Estructura porcentual de articulos científicos } \\
\text { según la fuente de indización }\end{array}$ \\
\hline $\begin{array}{l}\text { - Estructura porcentual de Programas docentes } \\
\text { según el nivel académico impartido }\end{array}$ & $\begin{array}{l}\text { - Estructura porcentual de la Producción cientifica } \\
\text { según el idioma de publicación }\end{array}$ \\
\hline $\begin{array}{l}\text { - Estructura porcentual de Matrícula de Alumnos por } \\
\text { año según el nivel cursado }\end{array}$ & $\begin{array}{l}\text { - Estructura porcentual de artículos cientificos según } \\
\text { el origen de la revista }\end{array}$ \\
\hline $\begin{array}{l}\text { Proyección de Crecimiento de Recursos Humanos } \\
\text { según el cargo que ocuparon para el } 2012\end{array}$ & $\begin{array}{l}\text { - Proyección de Crecimiento de la Producción } \\
\text { científica según la tipología documental }\end{array}$ \\
\hline $\begin{array}{l}\text { - Proyección de Crecimiento de Recursos Humanos } \\
\text { según el último grado obtenido para el } 2012\end{array}$ & $\begin{array}{l}\text { - Proyección de Crecimiento de la Producción } \\
\text { cientifica según el lugar de publicación }\end{array}$ \\
\hline $\begin{array}{l}\text { Proyección de Crecimiento de Proyectos de Investi- } \\
\text { gación según la tipología para el } 2012\end{array}$ & $\begin{array}{l}\text { - Proyección de Crecimiento de articulos científicos } \\
\text { según la fuente de indización }\end{array}$ \\
\hline $\begin{array}{l}\text { - Proyección de Crecimiento de Programas docentes } \\
\text { según el nivel académico impartido para el } 2012\end{array}$ & $\begin{array}{l}\text { - Proyección de Crecimiento de la Producción } \\
\text { cientifica según el idioma de publicación }\end{array}$ \\
\hline $\begin{array}{l}\text { - Proyección de Crecimiento de Matrícula de Alum- } \\
\text { nos por año según el nivel cursado para el } 2012\end{array}$ & $\begin{array}{l}\text { - Proyección de Crecimiento de artículos científicos } \\
\text { según el origen de la revista }\end{array}$ \\
\hline
\end{tabular}

Fuente: Propuesta de un indicador para medir el comportamiento del desarrollo disciplinar de las Ciencias Bibliotecológica y de la Información en instituciones académicas, Gorbea Portal, Piña Pozas.

\section{DISEÑO Y POBLACIÓN DE ESTUDIO}

Docentes de la Carrera de Licenciatura en Ciencias de la Información (LCI), Maestría en Ciencias de la Información (MCI) y la Especialización en Gestión Documental y Administración de Archivos de la Facultad Politécnica de la Universidad Nacional de Asunción, totalizando 59 personas. 
Han respondido al cuestionario 53 docentes, de los cuales 34 son docentes de pregrado y 14de la Maestríay 8 docentes de laEspecialización. Del total,3 no han completado el cuestionario, y 3 son docentes en la Licenciatura, así como en los cursos de postgrado.

El período de tiempo analizado en el desarrollo professional de los docentes es del 2009 al 2013.

\section{ANÁLISIS DE LOS RESULTADOS}

Tabla 3: Población en estudio por carrera y curso de postgrado

\begin{tabular}{|c|c|r|r|r|}
\hline $\begin{array}{c}\text { Curso } \\
\text { Licenciatura }\end{array}$ & $\begin{array}{c}\text { Cantidad de } \\
\text { Módulos/Asignaturas * }\end{array}$ & $\begin{array}{c}\text { Total de } \\
\text { Docentes }\end{array}$ & $\begin{array}{c}\text { Docentes que } \\
\text { completaron }\end{array}$ & $\begin{array}{c}\text { Docentes } \\
\text { LCI/MCI/Esp. }\end{array}$ \\
\hline Maestría & 61 & 35 & 34 & \\
\hline Especialización & 17 & 16 & 14 & $\mathbf{2}$ \\
\hline Totales & 10 & 8 & 8 & $\mathbf{1}$ \\
\hline
\end{tabular}

Fuente: Datos colectados para la investigación. Año 2014

En relación al apartado de Docentes LCI/MCI/Esp., hace referencia a que determinados docentes, enseñan en la carrera de grado, así como en los cursos del Programa de Postgrado: Especialización en Gestión Documental y Administración de Archivos, y La Maestría en Ciencias de la Información. La relación cantidad de asignaturas/docentes que se observa en la Licenciatura, disparidad que se da a efecto, que un profesor, enseña en más de una asignatura en la carrera de pregrado. 


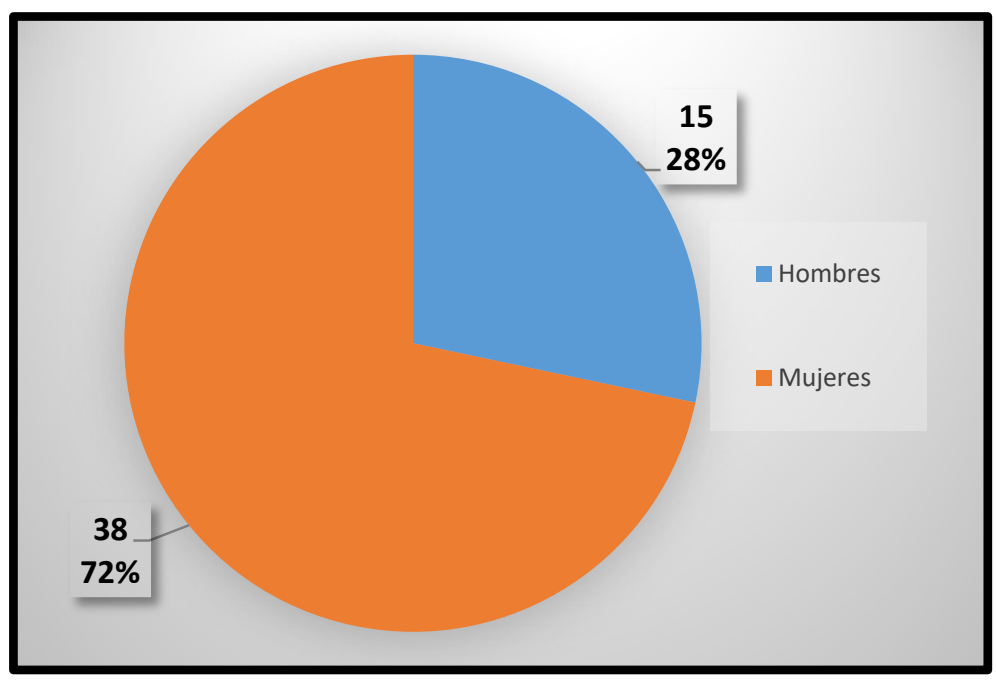

Gráfico 1. Porcentaje de docentes por género

Fuente: Datos colectados para la investigación. Año 2014.

El resultado que se presenta en el Gráfico 1 es muy común en esta especialidad, pues el gran porcentaje de docentes pertenece al gênero femenino, situación que también se observa cuando se analiza la población estudiantil de la carrera.

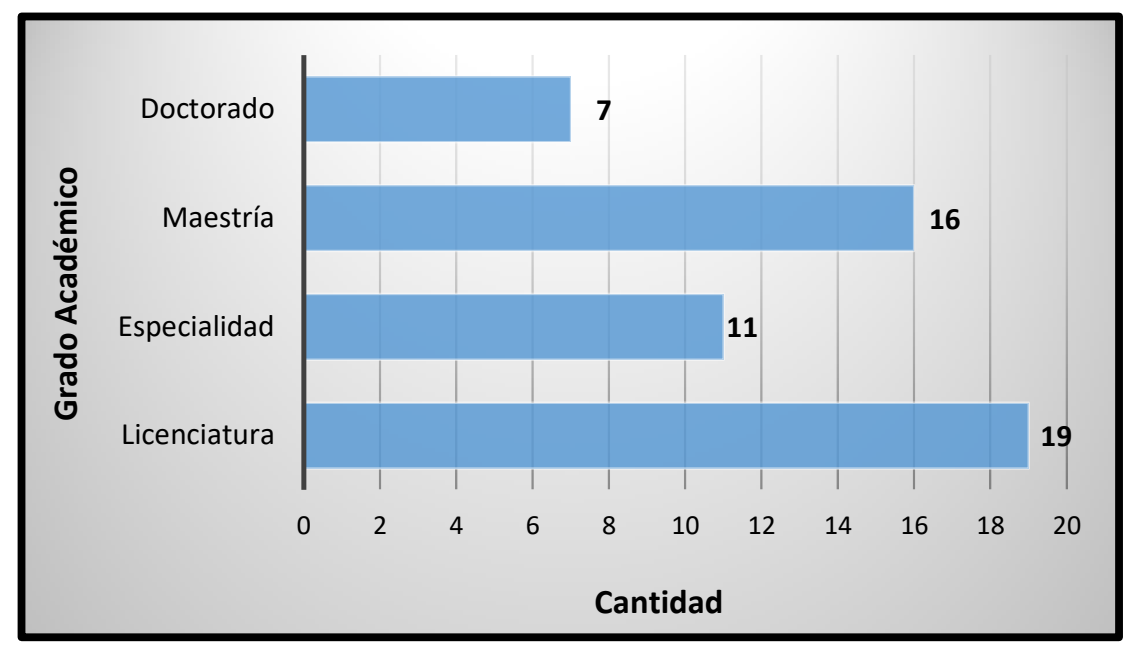

Gráfico 2. Porcentaje de docentes por género

Fuente: Datos colectados para la investigación. Año 2014

Como puede observarse en este Gráfico 2, al analizar la formación académica de los docentes, el nivel máximo de estudios predominante, es el de la Licenciatura. Éstos forman 
parte del plantel docente de la carrera de pregrado y para ejercer la docencia en este ámbito, no es exigência título de postgrado, como ocurre en otras universidades de la región. Los docentes que poseen títulos de estudios de postgrado, son aquellos que enseñan en los cursos de maestría, doctorado u otro postgrado, que en su mayoría son extranjeros.

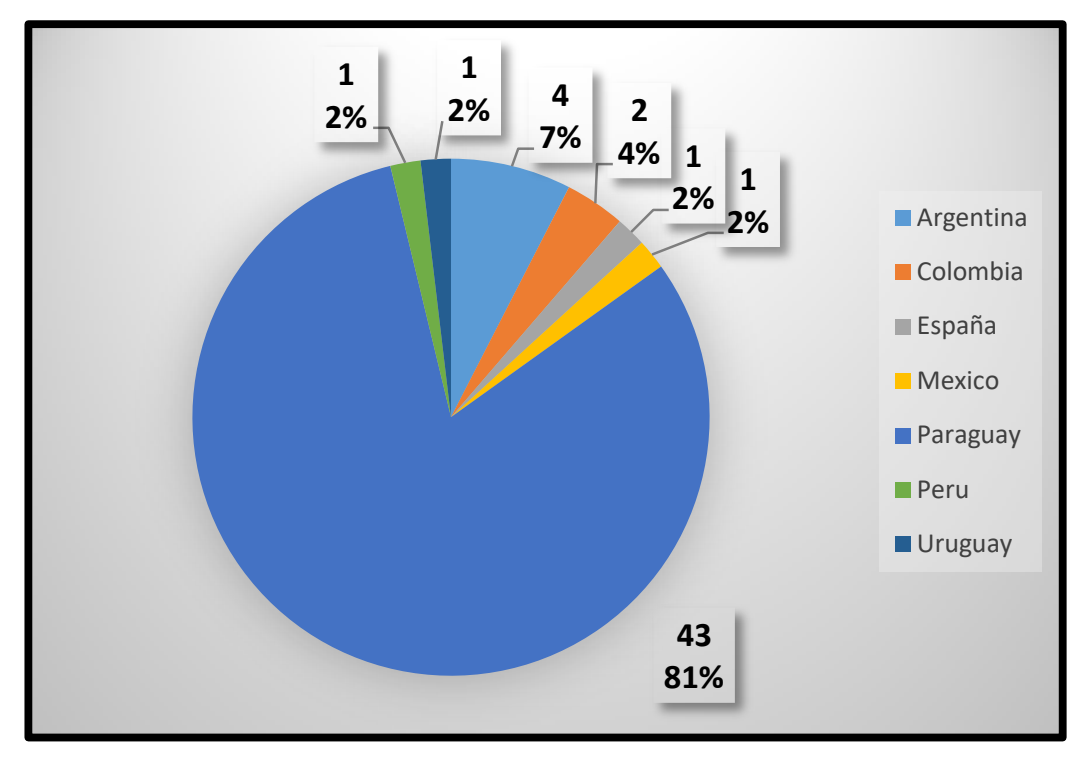

Gráfico 3. País de origen de los docentes

Fuente: Datos colectados para la investigación. Año 2014.

En el Gráfico 3, se muestra la procedencia de los docentes. Según estos datos, si nos referimos al total, encontramos que el $81 \%$ de los docentes son paraguayos y el $19 \%$ extranjeros, y los países con quienes se tienen una mayor vinculación son la Argentina y el Uruguay. Esto tiene su explicación por la cercanía regional y por la existencia del Programa de Movilidad Docente de la Asociación de Universidades del Grupo de Montevideo, conocida con la sigla AUGM. 


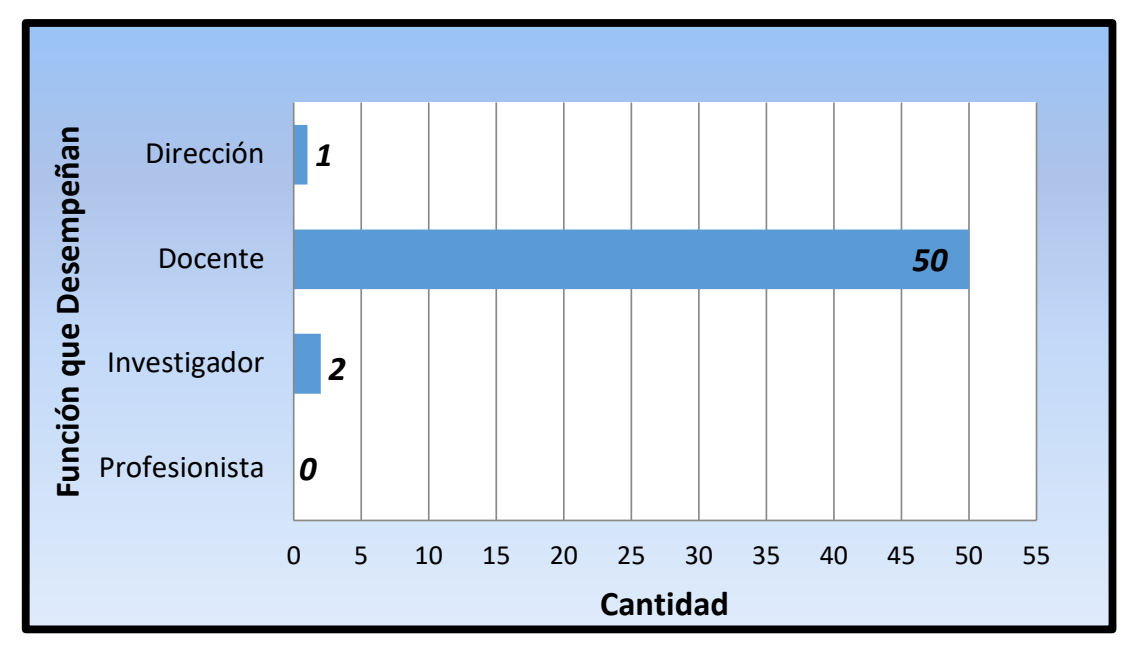

Gráfico 4. Cargo de los docentes

Fuente: Datos colectados para la investigación. Año 2014

Con respecto a las funciones de los $\mathbf{5 3}$ docentes encuestados, $\mathbf{5 0}$ declaran que son docentes, $\mathbf{1}$ ejerce el cargo de Director, en este caso, es la persona ocupa cargo de Directora de la Carrera de pregrado, 2 se definen como investigadores, que corresponden a investigadores del Grupo de Investigación en Ciencias de la Información, ambos con título de Dr. Sin embargo, esta función no implica la dedicación en forma exclusiva, es decir a tiempo completo para dichas actividades. En la Universidad, existe la figura del Docente Investigador con Dedicación Exclusiva: DITCoDE, pero, sin embargo, ningún profesional del área de las Ciencias de la Información, cuenta con este rubro, éste se enfoca más para las áreas de las ingenierías y tecnologías. 


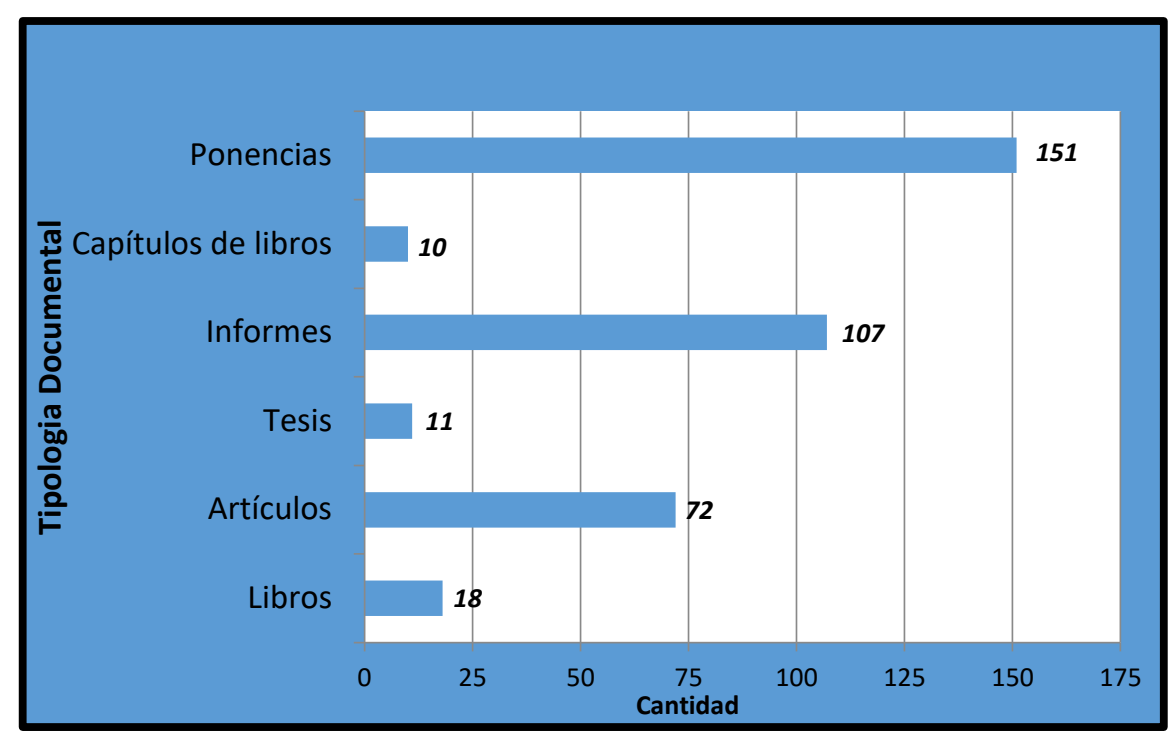

Gráfico 5. Producción de los docentes por tipología documental

Fuente: Datos colectados para la investigación. Año 2014

En cuanto a la tipología documental acumulada de las publicaciones de los docentes, se observa una gran cantidad de ponencias en eventos profesionales, asimismo se detalla una cantidad importante de informes, y ya en 3er lugar, posiciona los artículos en revistas. Se visualiza, una escasa publicación de libros, que se calcula un promedio de 3 libros, por docente durante el periodo de estúdio, 2009-2013. 


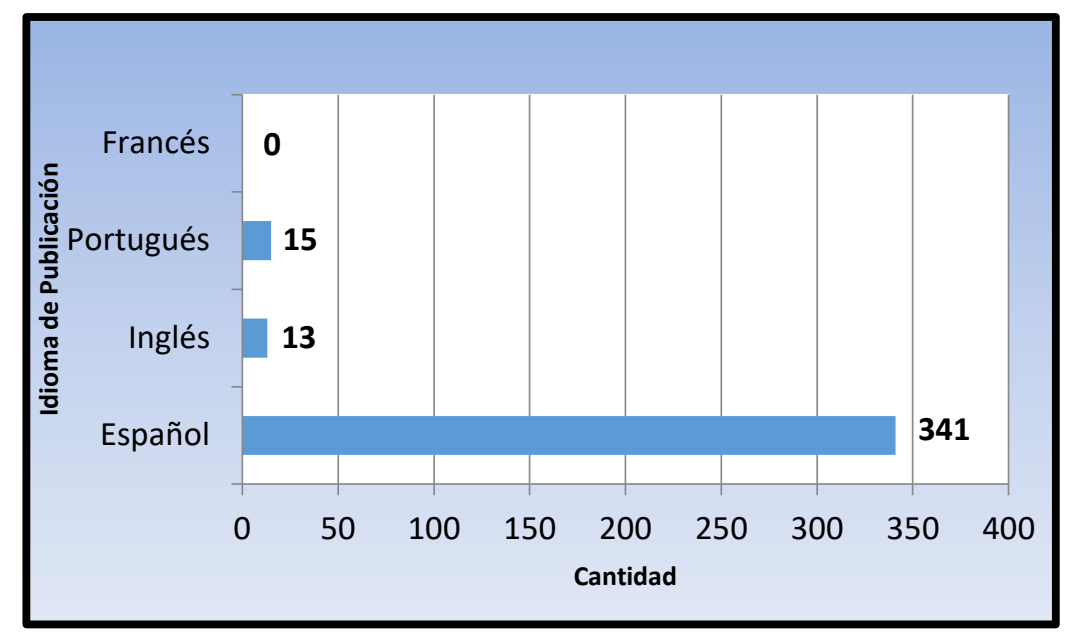

Gráfico 6. Producción de los docentes por idioma

Fuente: Datos colectados para la investigación. Año 2014.

En cuanto a los idiomas de publicación en el periodo de análisis 2009-2013, ha sido en castellano (341), seguido por el portugués, el inglés ocupa el 3er puesto y en francés no se há publicado ningún documento.

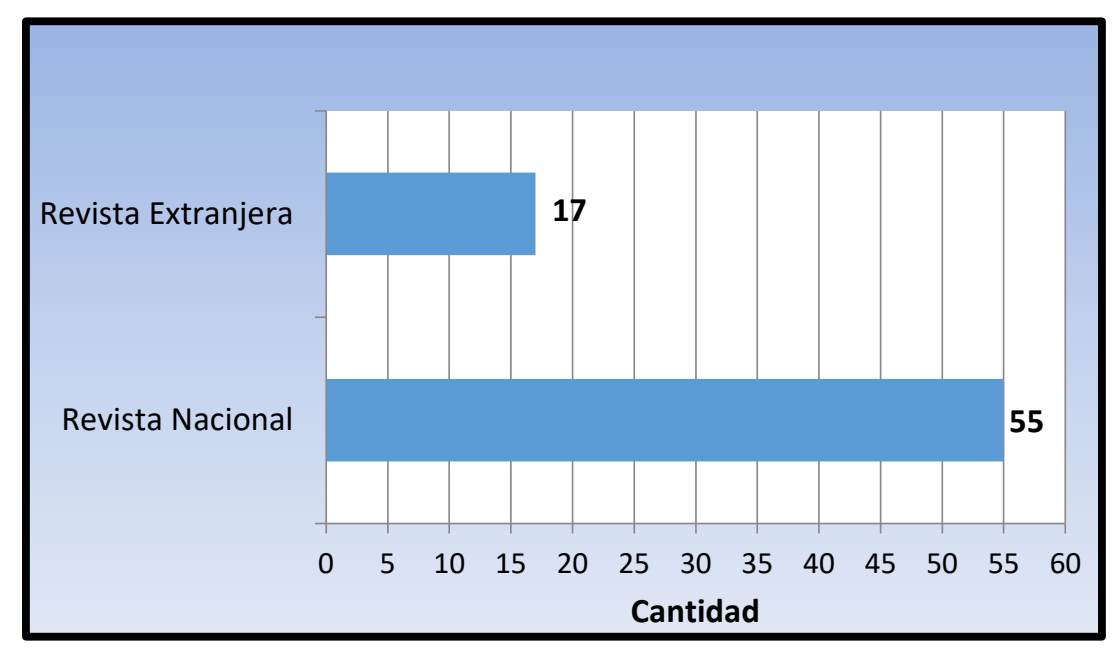

Gráfico 7. Dónde publican los artículos científicos los docentes

Fuente: Datos colectados para la investigación. Año 2014.

Las revistas nacionales son las fuentes donde más publican los artículos,en relación a las extranjeras. Se debe indicar que lãs revistas nacionales, no reúnen los estándares internacionales de factor de impacto, por lo que los investigadores paraguayos, a pesar de contar con una cantidad de producción de artículos, no tienen mucha presencia a nivel internacional. Esa diferencia, entre la publicación en revistas nacionales y extranjeras, se da 
por el incumplimiento de estándares editoriales de indexación en las más importantes bases de datos científicas internacionales.

\section{CONCLUSIONES}

Considerando que el artículo científico es el principal medio de comunicación de la ciencia, la producción científica -en general- de los docentes de la FP-UNA es baja: menos de 1 artículo por año y por docente. Tomando en cuenta la dimensión libros y capítulo de libros, la producción es aún menor. Se encontró que se genera 1 libro cada 8 años por docente.La dimensión "ponencias" también refleja baja participación: 1 ponencia cada 2 años por docente.

Del total de docentes (81\%), ejercen la docencia, la investigación y la extensión, pilares fundamentales de la educación superior.

Se observa según los resultados obtenidos, que la producción científica en el ámbito bibliotecológico es baja, posiblemente sea debido principalmente, por la falta de motivación hacía del desarrollo de la investigación científica, la baja cultura científica y deficiente formación en investigación de parte de los docentes.

En Paraguay desde el 2011, se instaló el Programa Nacional de Incentivo para Investigadores, PRONII, dependiente del Consejo Nacional de Ciencia y Tecnología, CONACYT, cuyo objetivo es la de fortalecer, consolidar y expandir la comunidad científica del país y establecer un sistema de incentivos económicos a los investigadores que estimule la dedicación a la producción científica en todas áreas del conocimiento. Este Programa, ha sido um gran paso para la ciencia nacional, que está dando sus resultados. 


\section{REFERÊNCIAS}

DUARTE MASI, Sergio. Existe centralización de la ciencia en Asunción-Central.ABC Color. Disponible en:http://www.abc.com.py/edicion-impresa/ciencia-y-tecnologia/existecentralizacion-de-la-ciencia-en-asuncion-central-1365562.html. 2015.

GORBEA PORTAL, Salvador.; PIÑA ROZAS, Maricela. Propuesta de un indicador para medir el comportamiento del desarrollo disciplinar de las Ciencias Bibliotecológica y de la Información en instituciones académicas. Investigación Bibliotecológica, v. 27, n. 60, mayo/agosto. 2003. p. 153-180.

SANCHO GIL, Juana. Docencia e investigación en la universidad: una profesión, dos mundos. Educar 28, p. 41-60, 2001.
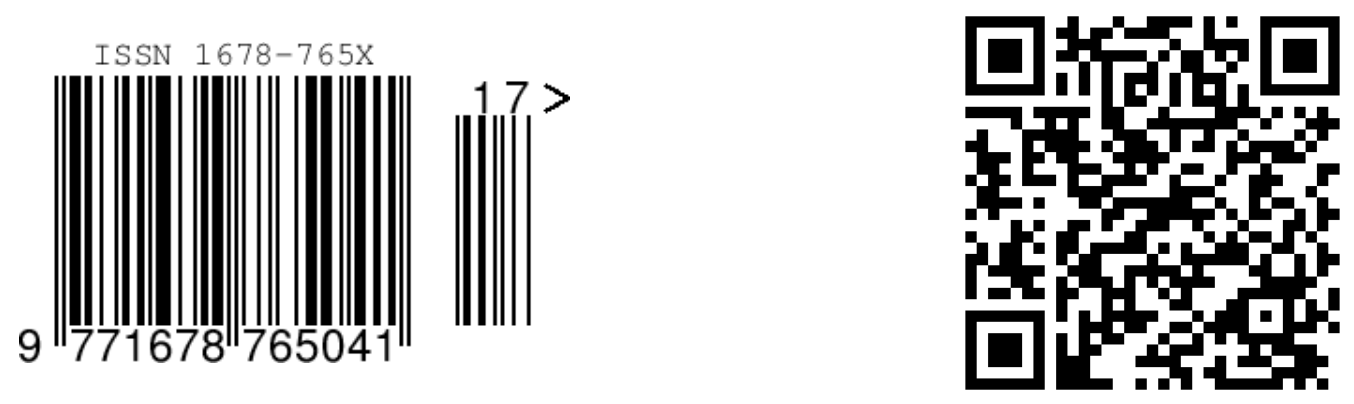